\title{
Theoretical Interpretation of Kerr Constants of Polymer Chains. n-Alkanes, Poly(oxyethylene glycol)s, and Poly(oxyethylene dimethyl Ether)s
}

\author{
Toshihide IshIKAwa and Kazuo NagaI \\ Government Industrial Research Institute, Osaka, \\ Midorigaoka 1, Ikeda, Osaka, Japan.
}

(Received December 15, 1970)

\begin{abstract}
Molar Kerr constants of $n$-alkanes, polyoxyethylene glycols (POEG), and polyoxyethylene dimethyl ethers (POEDE) are calculated on the basis of the bond additivity principle for dipole moments and polarizabilities and of the trans, gauche, and gauche prime, rotational-isomeric-state models. Theoretical values are compared with existing experimental data. It is found that agreement is very good with $n$ alkanes, but very poor with POEG. A new formulation of the molar Kerr constant is included.
\end{abstract}

KEY WORDS Kerr Constant / $n$-Alkanes / Poly(oxyethylene glycol)s /

Poly(oxyethylene dimethyl ether)s / Rotational-isomeric-state Model

We previously developed a general theory of Kerr constants of polymer molecules and applied it to the polyethylene chain of infinite length by taking into account realistic structures of polymer chains, i.e., fixed bond lengths and angles and hindered, interdependent, internal rotations. ${ }^{1}$ The theory was subsequently extended by Flory and Jernigan and by one of the authors to a chain of an arbitary bond sequence of finite length ${ }^{2-4}$ and a stereoregular chain of infinite length. ${ }^{4}$

Over the last two decades Le Fevre and collaborators have carried out extensive experimental and theoretical studies for a number of low- and high-molecular weight compounds in solution, and have achieved remarkable success in elucidation of conformations of molecules for lowmolecular weight compounds. ${ }^{5}$ Unfortunately, polymers have been exceptions on account of the lack of adequate theories. Having now the theories ${ }^{1-4}$ in hand, it is tempting to analyse experimental data. In this paper we treat $n$ alkanes, poly(oxyethylene glycol)s (POEG), and poly(oxyethylene dimethyl ether)s (POEDE). Relevant experimental data were reported by Stuart, Finck, and $\mathrm{Kuss}^{6}$ for the first and by Aroney, Levre, and Parkins ${ }^{7}$ for the second. In the course of this study we derived a new expression for the molar Kerr constant, with which the programming becomes somewhat easier than with previous expressions. ${ }^{2-4}$ This, together with its derivation, is also included here.

\section{THEORETICAL}

The molar Kerr constant $K_{m}$ for molecules, small or large, is expressed ${ }^{1}$ generally as

$$
K_{m}=\left(2 \pi N_{A} / 45 k^{2} T^{2}\right)\left(k T K_{p}+K_{d}\right)
$$

where $N_{A}$ is the Avogadro number, $k$ and $T$ have their usual meanings, and $K_{d}$ and $K_{p}$ are the dipole-moment and static-polarizability terms, respectively:

$$
\begin{aligned}
& K_{d}=3\left\langle\mathbf{u}^{T} \boldsymbol{\gamma} \mathbf{u}\right\rangle-\operatorname{Tr}\left\langle u^{2} \boldsymbol{\gamma}\right\rangle=3\left\langle\mathbf{u}^{T} \hat{\boldsymbol{\gamma}} \mathbf{u}\right\rangle \\
& \left.K_{p}=3 \operatorname{Tr}\left\langle\boldsymbol{\gamma} \boldsymbol{\gamma}^{\prime}\right\rangle-\langle\operatorname{Tr} \boldsymbol{\gamma})\left(\operatorname{Tr} \boldsymbol{\gamma}^{\prime}\right)\right\rangle=3 \operatorname{Tr}\left\langle\hat{\gamma} \boldsymbol{\gamma}^{\prime}\right\rangle
\end{aligned}
$$

In eq 2 and $3, \gamma$ and $\gamma^{\prime}$, respectively, are the optical- and static-polarizability tensors of a molecule; $\hat{\gamma}$ is the traceless form of $\gamma$; $\mathbf{u}$ is the dipole-monent vector with $u=|\mathbf{u}| ; \operatorname{Tr}$ and the superscript $T$, respectively, denote the trace and the transpose of a tensor; and the averages $\langle>$ refer to a molecule in its free state.

Consider a linear-polymer chain involving $n$ skeletal bonds indexed $1,2, \ldots, n$, successively from one end to the other. All bond lengths 


\section{T. IsHIKAWA and K. NAGAI}

and angles are assumed to be fixed and chain flexibility is therefore effected by rotation about skeletal bonds. The polymer chain is regarded as composed of $n$ groups of bonds, where group $i$ is assumed to involve all bonds except skeletal bond $i-1$, rigidly fixed to the framework determined by skeletal bonds $i-1$ and $i$. Each bond is referred to by bond $i \kappa$ which means the $\kappa$-th bond of group $i$. Skeletal bonds are dually indexed, i.e., skeletal bond $i$ or, say, bond $i 1$. As usual we assume the bond additivity for $\gamma$ :

$$
\begin{aligned}
& \boldsymbol{\gamma}=\sum_{i=1}^{n} \boldsymbol{\gamma}_{i} \\
& \left.\boldsymbol{\gamma}_{i}=\sum_{\kappa}\left[\alpha_{1}-\alpha_{2}\right)_{i \kappa} \boldsymbol{\mu}_{i \kappa} \boldsymbol{\mu}_{i_{\kappa}}^{T}+\alpha_{i \kappa 2} \mathbf{E}_{3}\right]
\end{aligned}
$$

where $\gamma_{i}$ is the tensor of group $i ; \alpha_{i \kappa 1}$ and $\alpha_{i \kappa 2}$ are the parallel and perpendicular components of polarizability of bond $i \kappa ;\left(\alpha_{1}-\alpha_{2}\right)_{i \kappa}=\alpha_{i \kappa 1}-\alpha_{i \kappa 2}$ is its anisotropy; $\mu_{i \kappa}$ is the unit vector along the bond, and $\mathbf{E}_{3}$ is the unit matrix of order three. In the present treatment we take advantage $^{2,3}$ of the simplicity achieved by using, as implied in eq 2 and 3 , the traceless tensor $\hat{\gamma}$ in place of $\gamma$ as previously used. ${ }^{4}$ We make $\gamma$ traceless by treating the tensor for every bond thus:

$$
\begin{gathered}
\hat{\boldsymbol{\gamma}}=\sum_{i=1}^{n} \hat{\boldsymbol{\gamma}}_{i}=\sum_{i=1}^{n} \sum_{\kappa} \hat{\boldsymbol{\gamma}}_{i \kappa} \\
\hat{\boldsymbol{\gamma}}=\sum_{\kappa} \hat{\boldsymbol{\gamma}}_{i \kappa}=\sum_{i}\left(\alpha_{1}-\alpha_{2}\right)_{i \kappa}\left(\boldsymbol{\mu}_{i \kappa} \boldsymbol{\mu}_{i \kappa}^{T}-\frac{1}{3} \mathbf{E}_{3}\right)
\end{gathered}
$$

where $\hat{\gamma}_{i}$ and $\hat{\gamma}_{i \kappa}$ are the traceless tensors of group $i$ and bond $i \kappa$.

We assume the bond additivity also for the dipole moment:

$$
\begin{aligned}
\mathbf{u} & =\sum_{i=1}^{n} \mathbf{u}_{i}=\sum_{i=1}^{n} \sum_{\kappa} \mathbf{u}_{i \kappa} \\
\mathbf{u}_{i} & =\sum_{\kappa} \mathbf{u}_{i \kappa}=\sum_{\kappa} m_{i \kappa} \boldsymbol{\beta}_{i \kappa}
\end{aligned}
$$

where $\mathbf{u}_{i}$ and $\mathbf{u}_{i \kappa}$ are the dipole-moment vectors of group $i$ and bond $i \kappa$, and $m_{i \kappa}=\left|\mathbf{u}_{i \kappa}\right|$.

At this stage we introduce a set of cartesian coordinate systems as follows., ${ }^{3,8}$ Coordinate system $i$ is attached to the plane determined by skeletal bonds $i-1$ and $i$ in the following way. The $x$-axis is in the direction of skeletal bond $i$ and the $y$-axis is in the plane of skeletal bonds $i-1$ and $i$, its positive direction making an acute angle with skeletal bond $i-1$. The $z$-axis is chosen so as to complete a right-handed system together with the $x$ - and $y$-axes. The internalrotation angle $\theta_{i}$ about skeletal bond $i$ is measured from the trans position toward the direction of the right-handed twist of the polymer chain. Two presentations $\mathbf{v}^{(i)}$ and $\mathbf{v}^{(i+1)}$ of components of a vector in coordinate systems $i$ and $i+1$ are correlated by

$$
\mathbf{v}^{(i)}=\mathbf{A}_{i} \mathbf{v}^{(i+1)}
$$

$$
\mathbf{A}_{i}=\left[\begin{array}{ccc}
\alpha & \beta & 0 \\
\beta \cos \theta & -\alpha \cos \theta & \sin \theta \\
\beta \sin \theta & -\alpha \sin \theta & -\cos \theta
\end{array}\right]_{i}
$$

where $\mathbf{A}_{i}$ is the transformation matrix; the subscript $i$ to the bracket is implied to apply to $\alpha, \beta$, and $\theta$ within the brackets as subscripts; and $\alpha_{i}=\cos \omega_{i}$ and $\beta_{i}=\sin \omega_{i}$ with $\omega_{i}$ being the angle between skeletal bonds $i$ and $i+1, i . e$, the supplementary bond angle.

The expression of a tensor or a vector depends on a coordinate system in which its components are measured. Let $\hat{\gamma}_{i}{ }^{(j)}$ be the presentation of $\hat{\gamma}_{i}$ in coordinate system $j$, and similarly with vectors. We write $\hat{\gamma}_{i}{ }^{(i)}$ especially as

$$
\hat{\gamma}_{i}^{(i)} \equiv \Delta \hat{\boldsymbol{a}}_{i}=\sum_{\kappa}\left(\alpha_{1}-\alpha_{2}\right)_{i \kappa}\left(\boldsymbol{\mu}_{i \kappa}^{(i)} \boldsymbol{\mu}_{i \kappa}^{(i) T}-\frac{1}{3} \mathbf{E}_{3}\right)
$$

and similarly

$$
\mathbf{u}_{i}^{(i)} \equiv \mathbf{m}_{i}=\sum_{\kappa} m_{i \kappa} \boldsymbol{\mu}_{i \kappa}^{(i)}
$$

If we define $\mathbf{u}_{i j}=\mathbf{u}_{i}+\mathbf{u}_{i+1}+\cdots+\mathbf{u}_{j-1}$ we can write $\mathbf{u}^{T} \hat{\gamma} \mathbf{u}$ as

$$
\begin{aligned}
\mathbf{u}^{T} \hat{\gamma} \mathbf{u}= & \sum_{i} \mathbf{u}^{T} \hat{\gamma}_{i} \mathbf{u}=\sum_{i}\left(\mathbf{u}_{1 i}+\mathbf{u}_{i, n+1}\right)^{T} \hat{\gamma}_{i}\left(\mathbf{u}_{1 i}+\mathbf{u}_{i, n+1}\right) \\
= & \sum_{i} \mathbf{u}_{1 i}^{T} \hat{\gamma}_{i} \mathbf{u}_{1 i}+\sum_{i} \mathbf{u}_{i, n+1}^{T} \hat{\gamma}_{i} \mathbf{u}_{i, n+1} \\
& +2 \mathbf{u}_{1 i}^{T} \hat{\gamma}_{i} \mathbf{u}_{i, n+1}
\end{aligned}
$$

The first sum $S_{1}$ in the rhs of eq 14 is written

$$
S_{1}=\sum_{i} \mathbf{u}_{1 i}^{T} \hat{\gamma}_{i} \mathbf{u}_{1 i}=\sum_{i}\left(\mathbf{u}_{1 i} \mathbf{u}_{1 i}^{T}\right)_{c}^{T} \hat{\gamma}_{i c}=\sum_{i}\left(\mathbf{u}_{1 i}^{T} \times \mathbf{u}_{1 i}^{T}\right) \hat{\gamma}_{i c}
$$

where the subscript $c$ denotes the column expression of a tensor, i.e.,

$$
\gamma_{c}=\left(\begin{array}{lllllllll}
\gamma_{11} & \gamma_{12} & \gamma_{13} & \gamma_{21} & \gamma_{22} & \gamma_{23} & \gamma_{31} & \gamma_{32} & \gamma_{33}
\end{array}\right)^{T}
$$

and $x$ denotes the direct product of two vectors 
or matrices. The direct product of two matrices $\mathbf{a}=\left\{a_{i j}\right\}$ and $\mathbf{b}=\left\{b_{i j}\right\}$ is defined by $\mathbf{a} \times \mathbf{b}=\left\{\mathbf{a} b_{i j}\right\}$. According to eq 10 we obtain ${ }^{9}$

$$
\begin{aligned}
& \mathbf{u}_{1 i}^{(i) T}=\mathbf{m}_{i-1}^{T} \mathbf{A}_{i-1}+\mathbf{m}_{i-2}^{T} \mathbf{A}_{i-2} \mathbf{A}_{i-1}+\cdots \\
& +\mathbf{m}_{1}^{T} \mathbf{A}_{1} \mathbf{A}_{2} \cdots \mathbf{A}_{i-1} \\
& =\left(\begin{array}{lll}
1 & \mathbf{0}
\end{array}\right) \mathbf{A}_{1}{ }^{\prime} \mathbf{A}_{2}{ }^{\prime} \cdots \mathbf{A}_{i-1}^{\prime}\left[\begin{array}{c}
\mathbf{0} \\
\mathbf{E}_{3}
\end{array}\right] \\
& \mathbf{A}_{k}{ }^{\prime}=\left[\begin{array}{cc}
1 & \mathbf{m}^{T} \mathbf{A} \\
\mathbf{0} & \mathbf{A}
\end{array}\right]_{k}
\end{aligned}
$$

Expressing now $\mathbf{u}_{1 i}^{T}$ and $\hat{\gamma}_{i c}$ in coordinate system $i$ we obtain

$$
\begin{aligned}
& S_{1}=\sum_{i}\left(\mathbf{u}_{1 i}^{(i) T} \times \mathbf{u}_{1 i}^{(i) T}\right) \Delta \hat{\boldsymbol{a}}_{i c} \\
& =\sum_{i}\left(\begin{array}{ll}
1 & 0
\end{array}\right)\left[\prod_{k=1}^{i-1}\left(\mathbf{A}_{k}{ }^{\prime} \times \mathbf{A}_{k}{ }^{\prime}\right)\right]\left[\begin{array}{c}
\mathbf{0} \\
\Delta \hat{\boldsymbol{a}}_{i c}^{*}
\end{array}\right]
\end{aligned}
$$

where $\Delta \hat{\boldsymbol{a}}_{i c}^{*}$ is the column expression of a matrix:

$$
\Delta \hat{\boldsymbol{\alpha}}_{c}{ }^{*}=\left(\mathbf{0}_{3,1} \Delta \hat{\boldsymbol{\alpha}}\right)_{c}
$$

with $\mathbf{0}_{3,1}$ being the null matrix of order $3 \times 1$.

Since

$$
\begin{aligned}
& \mathbf{u}_{i, n+1}^{(i)}=\mathbf{E}_{3} \mathbf{m}_{i}+\mathbf{A}_{i} \mathbf{m}_{i+1}+\cdots+\mathbf{A}_{i} \mathbf{A}_{i+1} \cdots \mathbf{A}_{n-1} \mathbf{m}_{n} \\
& =\left(\begin{array}{ll}
\mathbf{E}_{3} & \mathbf{0}
\end{array}\right) \mathbf{A}_{i}^{\prime \prime} \mathbf{A}_{i+1}^{\prime \prime} \cdots \mathbf{A}_{n}{ }^{\prime \prime}\left[\begin{array}{l}
\mathbf{0} \\
1
\end{array}\right] \\
& \mathbf{A}_{k}{ }^{\prime \prime}=\left[\begin{array}{cc}
\mathbf{A} & \mathbf{m} \\
\mathbf{0} & 1
\end{array}\right]_{k}
\end{aligned}
$$

the second sum $S_{2}$ in eq 14 is expressed as

$$
\begin{aligned}
S_{2} & =\sum_{i} \mathbf{u}_{i, n+1}^{T} \hat{\boldsymbol{\gamma}}_{i} \mathbf{u}_{i, n+1}=\sum_{i} \hat{\boldsymbol{\gamma}}_{i c}^{(i) T}\left(\mathbf{u}_{i, n+1}^{(i)} \times \mathbf{u}_{i, n+1}^{(i)}\right) \\
& =\sum_{\imath}\left(\begin{array}{ll}
\Delta \hat{\boldsymbol{a}}_{i c}^{\dagger T} & \mathbf{0}
\end{array}\right)\left[\prod_{l=i}^{n}\left(\mathbf{A}_{l}^{\prime \prime} \times \mathbf{A}_{l}{ }^{\prime \prime}\right)\right]\left[\begin{array}{l}
\mathbf{0} \\
1
\end{array}\right]
\end{aligned}
$$

with

$$
\Delta \hat{\boldsymbol{\alpha}}_{c}^{\dagger}=\left(\begin{array}{ll}
\Delta \hat{\boldsymbol{\alpha}} & \mathbf{0}_{3,1}
\end{array}\right)_{c}
$$

The third sum $S_{3}$ in eq 14 is expressed

$$
\begin{aligned}
& S_{3}=2 \sum_{i} \mathbf{u}_{1 i}^{T} \hat{\gamma}_{i} \mathbf{u}_{i, n+1} \\
& =2 \sum_{i}\left(\begin{array}{ll}
1 & 0
\end{array}\right) \mathbf{A}_{1}{ }^{\prime} \mathbf{A}_{2}{ }^{\prime} \cdots \mathbf{A}_{i-1}^{\prime}\left[\begin{array}{c}
\mathbf{0} \\
\mathbf{E}_{3}
\end{array}\right] \\
& \times \Delta \hat{\boldsymbol{\alpha}}_{i}\left(\mathbf{E}_{3} \quad \mathbf{0}\right) \mathbf{A}_{i}{ }^{\prime \prime} \mathbf{A}_{i+1}^{\prime \prime} \cdots \mathbf{A}_{n}{ }^{\prime \prime}\left[\begin{array}{l}
\mathbf{0} \\
1
\end{array}\right]
\end{aligned}
$$

The three terms $S_{1}, S_{2}$, and $S_{3}$ can be condensed into a single term as follows

$$
\begin{aligned}
\mathbf{u}^{T} \hat{\gamma} \mathbf{u} & =S_{1}+S_{2}+S_{3} \\
& =\sum_{i}\left(\begin{array}{ll}
1 & 0
\end{array}\right)\left[\prod_{k=1}^{i-1}\left(\mathbf{A}_{k}{ }^{\prime} \times \mathbf{A}_{k}{ }^{\prime}\right)\right]
\end{aligned}
$$

$$
\begin{aligned}
& \times \Delta \hat{\boldsymbol{\alpha}}_{i}{ }^{\ddagger}\left[\prod_{l=i}^{n}\left(\mathbf{A}_{l}{ }^{\prime \prime} \times \mathbf{A}_{l}{ }^{\prime \prime}\right)\right]\left[\begin{array}{l}
\mathbf{0} \\
1
\end{array}\right] \\
& \Delta \hat{\boldsymbol{\alpha}}^{\ddagger}=\left[\begin{array}{ccc}
\Delta \hat{\boldsymbol{\alpha}}_{c}^{\dagger T} & \mathbf{0} & \mathbf{0} \\
\mathbf{0} & 2 \Delta \hat{\boldsymbol{\alpha}} & \mathbf{0} \\
\mathbf{0} & \mathbf{0} & \Delta \hat{\boldsymbol{\alpha}}_{c}^{*}
\end{array}\right]
\end{aligned}
$$

It is easy to confirm that if $\Delta \hat{\boldsymbol{a}}_{i}^{\ddagger}$ in eq 26 is decomposed into $\Delta \boldsymbol{\alpha}_{i c}^{\dagger T}, 2 \Delta \hat{\boldsymbol{\alpha}}_{i}$ and $\Delta \boldsymbol{\alpha}_{i c}^{*}$, these terms yield $S_{2}, S_{3}$, and $S_{1}$, respectively. Summation $^{9}$ over $i$ in eq 26 yields

$$
\mathbf{u}^{T} \hat{\boldsymbol{\gamma}} \mathbf{u}=\left(\begin{array}{ll}
1 & \mathbf{0}
\end{array}\right)\left[\prod_{i=1}^{n}\left[\begin{array}{cc}
\mathbf{A}^{\prime} \times \mathbf{A}^{\prime} & \Delta \hat{\boldsymbol{a}}^{\ddagger}\left(\mathbf{A}^{\prime \prime} \times \mathbf{A}^{\prime \prime}\right) \\
\mathbf{0} & \mathbf{A}^{\prime \prime} \times \mathbf{A}^{\prime \prime}
\end{array}\right]_{i}\right]\left[\begin{array}{l}
\mathbf{0} \\
1
\end{array}\right]
$$

The size of the matrix in eq 28 is $32 \times 32$. For computational purposes it is obviously desirable to decrease the size of matrices as much as possible. In previous treatments ${ }^{1,4,10,11}$ this was achieved by utilizing the fact that $\hat{\gamma}$ is a symmetric tensor and its independent components are only six. Fortunately a similar procedure has been found possible in this case. We rewrite eq 26 as

$$
\begin{aligned}
& \mathbf{u}^{T} \hat{\boldsymbol{\gamma}} \mathbf{u}=\sum_{i}\left(\begin{array}{ll}
\mathbf{1} & \mathbf{0}
\end{array}\right) \mathbf{U}_{1}\left\{\prod_{k=1}^{i-1}\left[\mathbf{U}_{1}{ }^{T}\left(\mathbf{A}_{k}{ }^{\prime} \times \mathbf{A}_{k}{ }^{\prime}\right) \mathbf{U}_{1}\right]\right\} \\
& \quad \times \mathbf{U}_{1}{ }^{T} \Delta \hat{\boldsymbol{a}}_{i}{ }^{\ddagger} \mathbf{U}_{1}\left\{\prod_{l=1}^{n}\left[\mathbf{U}_{1}{ }^{T}\left(\mathbf{A}_{l}{ }^{\prime \prime} \times \mathbf{A}_{l}{ }^{\prime \prime}\right) \mathbf{U}_{1}\right]\right\} \mathbf{U}_{1}{ }^{T}\left[\begin{array}{l}
\mathbf{0} \\
1
\end{array}\right]
\end{aligned}
$$

where $\mathbf{U}_{1}$ is an orthogonal matrix: $\mathbf{U}_{1} \mathbf{U}_{1}{ }^{T}=\mathbf{E}$. Proper choice of $\mathbf{U}_{1}$ permits us to separate significant elements from zero elements so that the latter can be easily deleted. A form of $\mathbf{U}_{1}$ is found to be

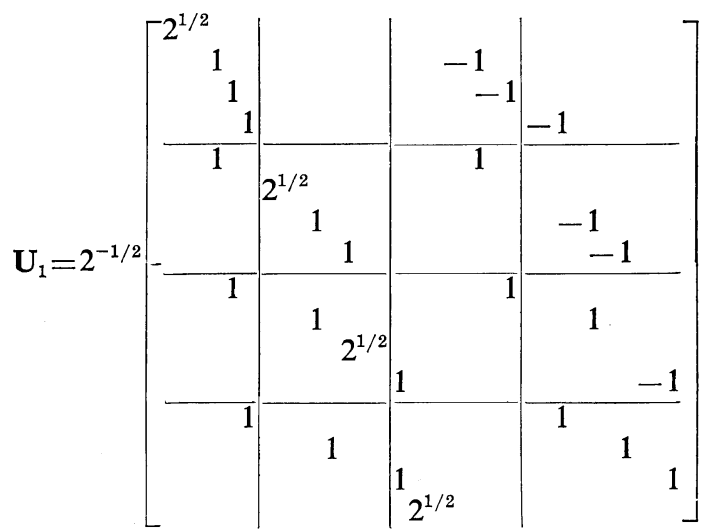


where elements not explicitly shown are zero. With this $\mathbf{U}_{1}$ we find

$$
\begin{aligned}
& \mathbf{U}_{1}^{T}\left(\mathbf{A}^{\prime} \times \mathbf{A}^{\prime}\right) \mathbf{U}_{1}=\left[\begin{array}{cc}
\tilde{\mathbf{A}}^{\prime} & \mathbf{0} \\
\mathbf{0} & \tilde{\mathbf{A}}_{22}^{\prime}
\end{array}\right], \\
& \mathbf{U}_{1}^{T}\left(\mathbf{A}^{\prime \prime} \times \mathbf{A}^{\prime \prime}\right) \mathbf{U}_{1}=\left[\begin{array}{cc}
\tilde{\mathbf{A}}^{\prime \prime} & \mathbf{0} \\
\mathbf{0} & \widetilde{\mathbf{A}}_{22}^{\prime \prime}
\end{array}\right]
\end{aligned}
$$

and

$$
\mathbf{U}_{1}^{T} \Delta \hat{\boldsymbol{a}}^{\ddagger} \mathbf{U}_{1}=\left[\begin{array}{ll}
\Delta \hat{\boldsymbol{\alpha}} & \Delta \hat{\boldsymbol{\alpha}}_{12} \\
\Delta \hat{\boldsymbol{\alpha}}_{21} & \Delta \hat{\boldsymbol{\alpha}}_{22}
\end{array}\right]
$$

and $\left(\begin{array}{ll}1 & \mathbf{0}\end{array}\right) \mathbf{U}_{1}=\left(\begin{array}{ll}1 & 0\end{array}\right)$ and

$$
\mathbf{U}_{1}^{T}\left[\begin{array}{l}
\mathbf{0} \\
1
\end{array}\right]=\left[\begin{array}{l}
\mathbf{0}_{9,1} \\
1 \\
\mathbf{0}_{6,1}
\end{array}\right]
$$

where $\tilde{\mathbf{A}}^{\prime}, \widetilde{\mathbf{A}}^{\prime \prime}$, and $\Delta \tilde{\boldsymbol{\alpha}}$ are square matrices of order ten. After substitution of these relations into eq 29 we see that, of the ensuing matrices, all rows and columns exceeding the tenth contribute nothing to $\mathbf{u}^{T} \hat{\gamma} \mathbf{u}$. Deleting these redundant rows and columns we obtain

$$
\begin{gathered}
\mathbf{u}^{T} \hat{\boldsymbol{\gamma}} \mathbf{u}=\sum_{i}\left(\begin{array}{ll}
1 & \mathbf{0}
\end{array}\right)\left[\prod_{k=1}^{i-1} \tilde{\mathbf{A}}_{k}^{\prime}\right] \Delta \tilde{\boldsymbol{\alpha}}_{i}\left[\prod_{l=i}^{n} \tilde{\mathbf{A}}_{l}^{\prime \prime}\right]\left[\begin{array}{l}
\mathbf{0} \\
1
\end{array}\right] \\
=\left(\begin{array}{ll}
1 & \mathbf{0}
\end{array}\right) \mathbf{D}_{1} \mathbf{D}_{2} \cdots \mathbf{D}_{n}\left[\begin{array}{l}
\mathbf{0} \\
1
\end{array}\right] \\
\mathbf{D}_{i}=\left[\begin{array}{cc}
\tilde{\mathbf{A}}^{\prime} & \Delta \tilde{\boldsymbol{\alpha}} \tilde{\mathbf{A}}^{\prime \prime} \\
\mathbf{0} & \tilde{\mathbf{A}}^{\prime \prime}
\end{array}\right]_{i}
\end{gathered}
$$

The order of $\mathbf{D}_{i}$ is 20 .

The polarizability term was derived previously by Flory and Jernigan. ${ }^{2,3}$ It can be calculated by a mean similar to, but simpler than, that for the dipole-moment term. The only important fact is the following. According to the definition of a tensor we have $\boldsymbol{\gamma}^{(i-1)}=$ $\mathbf{A}_{i-1} \boldsymbol{\gamma}^{(i)} \mathbf{A}_{i-1}^{T}$. As may be readily confirmed, this relation is equivalent to $\boldsymbol{\gamma}_{c}{ }^{(i-1)}=\left(\mathbf{A}_{i-1} \times \mathbf{A}_{i-1}\right) \boldsymbol{\gamma}_{c}{ }^{(i)}$. Applying the method of reduction of sizes of matrices to Flory and Jernigan's result, we obtain

$$
\begin{aligned}
& \operatorname{Tr} \hat{\gamma} \gamma^{\prime}=\left(\begin{array}{lll}
1 & \mathbf{0}
\end{array}\right) \mathbf{P}_{1} \mathbf{P}_{2} \ldots \mathbf{P}_{n}\left[\begin{array}{l}
0 \\
1
\end{array}\right]
\end{aligned}
$$

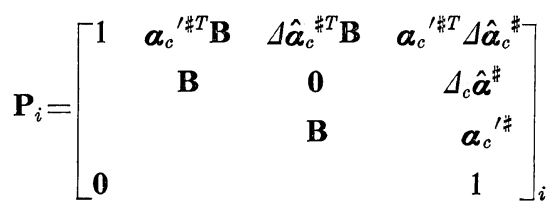

Here $\mathbf{B}$ is obtained from $\mathbf{A} \times \mathbf{A}$ by using an orthogonal matrix $\mathbf{U}_{2}$ :

$$
\mathbf{U}_{2}{ }^{T}(\mathbf{A} \times \mathbf{A}) \mathbf{U}_{2}=\left[\begin{array}{cc}
\mathbf{B} & \mathbf{0} \\
\mathbf{0} & \mathbf{B}_{22}
\end{array}\right]
$$

The order of $\mathbf{B}$ is six. A form of $\mathbf{U}_{2}$ is found to be

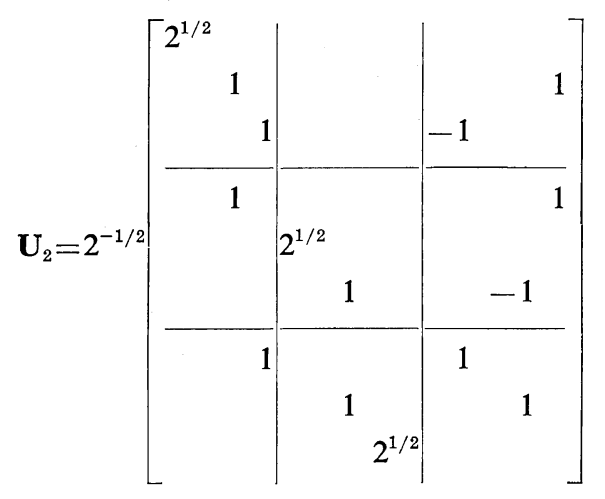

Then, $\Delta \hat{\boldsymbol{a}}_{c}^{\#}$ is given by

$$
\begin{aligned}
& \Delta \hat{\boldsymbol{\alpha}}_{c}^{\#} \\
& \quad=\left(\Delta \hat{\alpha}_{11}, 2^{1 / 2} \Delta \hat{\alpha}_{12}, 2^{1 / 2} \Delta \hat{\alpha}_{13}, \Delta \hat{\alpha}_{22}, 2^{1 / 2} \Delta \hat{\alpha}_{23}, \Delta \hat{\alpha}_{33}\right)^{T}
\end{aligned}
$$

where $\Delta \hat{\alpha}_{k l}$ is the $k, l$ element of $\Delta \hat{\hat{\alpha}}$ in eq 12 . $\boldsymbol{a}_{c}{ }^{\prime \#}$, which refers to the static polarizability, is defined anologous to $\Delta \hat{\boldsymbol{a}}_{c}^{\#}$ but from a non-traceless form for $\gamma_{i}{ }^{\prime}$ like eq 5 for $\gamma_{i}$. The traceless form $\boldsymbol{\alpha}_{c}{ }^{\prime \prime}$ can equally be used in place of $\boldsymbol{\alpha}_{c}{ }^{\prime}$, as is apparent from eq 3.

Averaging of $\mathbf{u}^{T} \hat{\gamma} \mathbf{u}$ and $\operatorname{Tr} \hat{\gamma} \gamma^{\prime}$ can be performed as usual with recourse to the rotationalisomeric-state approximation, according to which $\theta_{i}$ is assumed to be in either $s$ discrete values, or "states", $\theta^{(1)}, \theta^{(2)}, \ldots, \theta^{(s)}$, instead of continuum values. These states may be equal or different for different bonds both in number and in location. The conformational partition function of the polymer chain is expressed ${ }^{12}$ as

$$
Z=\mathbf{e}_{s}{ }^{T} \mathbf{p}_{1} \mathbf{p}_{2} \cdots \mathbf{p}_{n} \mathbf{e}_{s}
$$

where $\mathbf{e}_{s}$ is a $s$-dimensional vector defined by $\mathbf{e}_{s}=\left(\begin{array}{llll}1 & 0 & \ldots 0\end{array}\right)^{T} ; \mathbf{p}_{i}(i=1, \ldots, n-1)$ is the statistical-weight matrix for skeletal bonds $i$ and $i+1$; and $\mathbf{p}_{n}=\mathbf{E}_{s}$. A form of $\mathbf{p}_{i}$ is given ${ }^{12}$ by

$$
\mathbf{p}_{i}=\exp \left\{-\left[E_{i}\left(\theta_{i}{ }^{(k)}\right)+E_{i}\left(\theta_{i}{ }^{(k)}, \theta_{i+1}^{(l)}\right)\right] / R T\right\}
$$

where $R$ is the gas constant, and $E_{i}\left(\theta_{i}\right)$ and $E_{i}\left(\theta_{i}, \theta_{i+1}\right)$ are the one-bond and two-bond in- 
teraction energies. It is assumed that $E_{1}\left(\theta_{1}\right)=$ $E_{1}\left(\theta_{1}, \theta_{2}\right)=E_{n-1}\left(\theta_{n-1}, \theta_{n}\right)=0$. Actual constructions of $\mathbf{p}_{i}$ will be illustrated in the following sections. The above formulation ${ }^{12}$ of $Z$ is slightly different from that given previously, ${ }^{4,13}$ but is more convenient for construction of $\mathbf{p}_{i}$ and other matrices which follow.

We introduce the pseudo-diagonal supermatrices $\|\mathbf{D}\|$ and $\|\mathbf{P}\|$ whose $k$-th diagonal elements are $\mathbf{D}\left(\theta^{(k)}\right)$ and $\mathbf{P}\left(\theta^{(k)}\right)$, respectively. We then have

$$
\begin{aligned}
& \left\langle\mathbf{u}^{T} \hat{\boldsymbol{\gamma}} \mathbf{u}\right\rangle=\boldsymbol{Z}^{-1}\left(\mathbf{e}_{\mathbf{s}}{ }^{T} \quad \mathbf{0}\right)\left[\prod_{i=1}^{n}\left\|\mathbf{D}_{i}\right\|\left(\mathbf{E}_{20} \times \mathbf{D}_{i}\right)\right]\left[\begin{array}{c}
\mathbf{0} \\
\mathbf{e}_{s}
\end{array}\right] \\
& \langle\operatorname{Tr} \hat{\gamma} \boldsymbol{\gamma}\rangle=Z^{-1}\left(\mathbf{e}_{s}{ }^{T} \quad \mathbf{0}\right)\left[\prod_{i=1}^{n}\left\|\mathbf{P}_{i}\right\|\left(\mathbf{E}_{14} \times \mathbf{p}_{i}\right)\right]\left[\begin{array}{c}
0 \\
\mathbf{e}_{s}
\end{array}\right]
\end{aligned}
$$

The optical anisotropy defined by

$$
\left\langle(\Delta \gamma)^{2}\right\rangle=\frac{3}{2}\left\langle\operatorname{Tr} \hat{\gamma}^{2}\right\rangle
$$

is obtained from eq 42 with $\gamma^{\prime}$ equated to $\hat{\gamma}$. It can, however, be expressed in terms of matrices of lower size by the ensuing symmetry., ${ }^{3,4}$

Explicit expressions for $\widetilde{\mathbf{A}}^{\prime}$ and $\mathbf{B}$ were not given; they are unnecessary, being constructed numerically within a computer according to eq 31 and 36. In the previous treatment $t^{4}$ the dipole-moment and polarizability terms were condensed into a single term. This was not possible with the present scheme. Separate calculations of the two terms are convenient in cases where contributions of these terms to $K_{m}$ are needed to know, or where programs are used differently, $i . e$., to calculate the stress-optical coefficient and the optical anisotropy. It is found that the present scheme makes programming easier, as manifested in a shorter main routine, but requires more working memories and computing time, compared with previous treatments. ${ }^{2-4}$

\section{n-ALKANES}

We adopt the familiar three-state rotationalisometric model: trans $\left(\theta^{(T)}=0^{\circ}\right)$, gauche $\left(\theta^{(G)}=\right.$ $\left.120^{\circ}\right)$, and gauche prime $\left(\theta^{(G)}=-120^{\circ}\right) .^{14-16}$ All bond angles are assumed to be tetrahedral, and considerable simplification results thereby. The $G G^{\prime}$ and $G^{\prime} G$ conformations for neighboring bonds are eliminated. Putting $\mathbf{p}_{1}=\mathbf{p}_{2}=\cdots=$ $\mathbf{p}_{n-1}=\mathbf{p}$ and $\mathbf{p}_{n}=\mathbf{E}_{3}$ we obtain for the $n$-alkane chain consisting of $n \mathrm{C}-\mathrm{C}$ bonds

$$
\begin{gathered}
\mathbf{Z}=\mathbf{e}_{3}{ }^{T} \mathbf{p}^{n-1} \mathbf{p}_{n} \mathbf{e}_{3} \\
\mathbf{p}=\left[\begin{array}{lll}
1 & 1 & 1 \\
\sigma & \sigma & 0 \\
\sigma & 0 & \sigma
\end{array}\right]
\end{gathered}
$$

with $\sigma=\exp \left(-E_{G} / R T\right)$ where $E_{G}$ is the energy of the $G$ state relative to the $T$ state. According to the definition given by eq 40 , all elements of $\mathbf{p}_{1}$ are unity, but, $\mathbf{p}_{1}$ was modified, being equated to $\mathbf{p}$. It is apparent that this modification simplifies $Z$ without error.

The bond dipole moments $m_{\mathrm{C}-\mathrm{C}}$ and $m_{\mathrm{C}-\mathrm{H}}$ are taken to be zero in view of virtually zero observed dipole moments of $n$-alkanes. ${ }^{17}$ Hence the polarizability term alone needs to be considered, and the treatment is very close to that of the optical anisotropy. ${ }^{18}$ With the assumed tetrahedral symmetry for bond angles, it can be regarded that $\mathrm{C}-\mathrm{C}$ bonds were to have the effective anisotropy

$$
\Gamma_{\mathrm{C}-\mathrm{C}}=\left(\alpha_{1}-\alpha_{2}\right)_{\mathrm{C}-\mathrm{C}}-2\left(\alpha_{1}-\alpha_{2}\right)_{\mathrm{C}-\mathrm{H}}
$$

while $\mathrm{C}-\mathrm{H}$ bonds had no anisotropy. ${ }^{19,20}$ Static bond polarizabilities are assumed to be greater than optical ones by a factor of 1.1, following

Table I. Effective bond optical anisotropies

\begin{tabular}{ccccccc}
\hline Author & Denbigh $^{21}$ & $\begin{array}{c}\text { Clement \& } \\
\text { Bothorel }^{22}\end{array}$ & Le Fevre & $\begin{array}{c}\text { Bunn \& } \\
\text { Daubeny }\end{array}$ & Feurche $^{24}$ & $\begin{array}{c}\text { Le Fevre } \\
(\text { modified })^{\mathrm{a}}\end{array}$ \\
\hline$\Gamma_{\mathrm{C}-\mathrm{C}}\left(\AA^{3} \AA^{3}\right)$ & 1.44 & $0.81,0.87$ & 0.72 & 0.29 & - & 0.81 \\
$\Gamma_{\mathrm{C}-\mathrm{O}}\left(\AA^{3}\right)$ & 0.77 & - & 0.42 & - & 1.0 & 0.23 \\
\hline
\end{tabular}

a With the assumptions of tetrahedral symmetry and $\Gamma^{\prime}=1.1 \Gamma,{ }_{m} K\left(=K_{m} / 9\right)$ for the chair-form paraldehyde with all methyl groups in the equatorial conformation is given by ${ }_{m} K=\left(4 \pi N_{A} / 405 k T\right)[1.1$ $\left.\left(\Gamma_{\mathrm{C}-\mathrm{C}}+2 \Gamma_{\mathrm{C}-\mathrm{O}}\right)^{2}-4 m_{\mathrm{C}-\mathrm{O}}{ }^{2}\left(\Gamma_{\mathrm{C}-\mathrm{C}}+2 \Gamma_{\mathrm{C}-\mathrm{O}}\right) / k T\right] . \quad \Gamma_{\mathrm{C}-\mathrm{O}}=0.23 \AA^{3}$ was obtained from the observed value ${ }_{m} K=$ $-57 \times 10^{-12}$ (C. G.S. $)^{25}$ by assuming $m_{\mathrm{C}-\mathrm{O}}=1.0 \mathrm{D}$ and $\Gamma_{\mathrm{C}-\mathrm{C}}=0.81 \AA^{3}$, based on the above expression. 
Table II. Calculated values of $\Lambda$ (in eq 47) and observed molar Kerr constants for $n$-alkanes

\begin{tabular}{rrrrr}
\hline$n$ & \multicolumn{3}{c}{$A$} & $\begin{array}{l}K_{m} \times 10^{12} \\
(\text { C.G.S. })^{\mathrm{a}}\end{array}$ \\
\cline { 2 - 5 } & \multicolumn{1}{c}{$20^{\circ} \mathrm{C}$} & \multicolumn{1}{c}{$50^{\circ} \mathrm{C}$} & \multicolumn{1}{c}{$80^{\circ} \mathrm{C}$} & \\
\hline 2 & 2.667 & 2.667 & 2.667 & \\
3 & 5.540 & 5.385 & 5.253 & \\
4 & 7.505 & 7.255 & 7.041 & 10.089 \\
5 & 10.969 & 10.486 & 10.083 & 15.264 \\
6 & 13.556 & 12.894 & 12.345 & 19.206 \\
7 & 17.207 & 16.250 & 15.468 & 23.931 \\
8 & 20.125 & 18.933 & 17.966 & 27.918 \\
9 & 23.814 & 22.292 & 21.069 & 32.895 \\
10 & 26.914 & 25.123 & 23.693 & \\
11 & 30.584 & 28.447 & 26.753 & 45.297 \\
12 & 33.793 & 31.367 & 29.452 & \\
13 & 37.429 & 34.650 & 32.469 & \\
14 & 40.708 & 37.629 & 35.219 & 56.556 \\
15 & 44.310 & 40.875 & 38.201 & 72.585 \\
16 & 47.636 & 43.892 & 40.985 & \\
17 & 51.207 & 47.111 & 43.940 & \\
18 & 54.568 & 50.157 & 46.749 & \\
\hline
\end{tabular}

a Converted from data of Stuart, et al. ${ }^{6}$

Le Fevre, ${ }^{5}$ and hence $\Gamma_{\text {C.C }}^{\prime}=1.1 \Gamma_{\text {C.C. }}$. This assumption will be made throughout this paper. Some literature values ${ }^{5,21-25}$ for $\Gamma_{\mathrm{C}-\mathrm{C}}$ are given in Table I. The polarizability parameters can be factored out in the expression for $K_{m}$, i.e.,

$$
K_{m}=\left(2 \pi N_{A} / 45 k T\right) \Gamma_{\mathrm{C}-\mathrm{C}} \Gamma^{\prime}{ }_{\mathrm{C}-\mathrm{C}} \Lambda
$$

where $A$ is a quantity dependent only on geometrical and statistical-weight parameters.

Calculated values of $\Lambda$ for $E_{G}=0.8 \mathrm{kcal} / \mathrm{mol}$ and $T=20,50$, and $80^{\circ} \mathrm{C}$ are given in Table II. ${ }^{*}$ Values of $K_{m}$ calculated by using these $\Lambda$ and various $\Gamma_{\mathrm{C}-\mathrm{C}}$ are plotted against $n$ in Figure 1.

Stuart, Finck, and $\mathrm{Kuss}^{6}$ carried out Kerreffect measurements for $n$-alkanes as pure liquids and in part as solutions with petroleum ether as a solvent. Unfortunately, details of experimental conditions were not given. Values of $K_{m}$ converted $^{* *}$ from their data on pure liquids

* The validity of the program was checked by the reproducibility, on the $K_{p}$ term, of the optical anisotropy of the chair-form cyclohexane, $(\Delta \gamma)^{2}=$ $\left.4 \Gamma_{\mathrm{C}-\mathrm{C}}\right)^{2}, 26$ and, on the $K_{d}$ term, of asymptotic values at the limit of infinite chain length. ${ }^{27}$

** Le $\mathrm{Fevre}^{5}$ defined the molar Kerr constant for pure liquids as

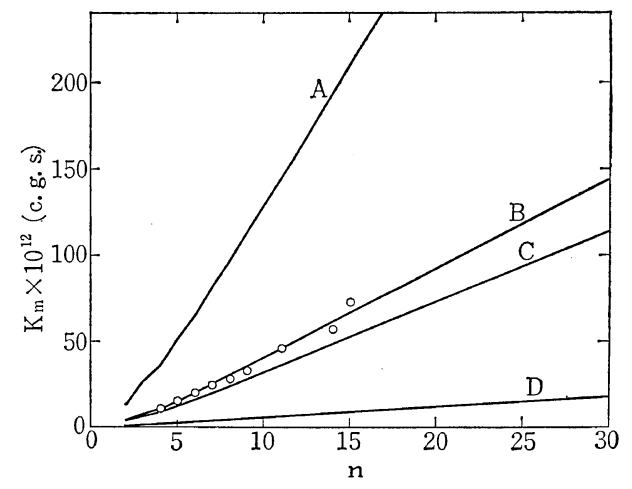

Figure 1. $K_{m}$ vs. $n$ for $n$-alkanes. Curves are calculated at $20^{\circ} \mathrm{C}$ using $E_{G}=0.8 \mathrm{kcal} / \mathrm{mol}$ and $\Gamma_{\mathrm{C}-\mathrm{C}}=$ 1.44 (A), $0.81(\mathrm{~B}), 0.72(\mathrm{C})$, and $0.29(\mathrm{D})$, all in $\AA^{3}$. Circles are experimental data for pure liquid n-alkanes by Stuart, et al. ${ }^{6}$

are shown by circles in Figure 1 and included in Table II. Necessary data of density, dielectric constant, and refractive index were taken from Landolt-Börnstein. ${ }^{28}$ Some results on longer alkanes as solutions were given by Stuart, et al., ${ }^{6}$ but cannot be converted to $K_{m}$ because of the lack of pertinent data.

Good agreement between theory and experiment is achieved if $\Gamma_{\mathrm{C}-\mathrm{C}}=0.81 \AA$ is used in pair with $E_{G}=0.8 \mathrm{kcal} / \mathrm{mol}$. The same situation was met in the theoretical analysis of related quantities, the optical anisotropy of $n$-alkanes in carbon tetrachloride solutions ${ }^{18}$ and the stress-optical coefficient of polyethylene networks swollen with decalin. ${ }^{29}$ Concerning the former quantity, a deviation was observed for $n$-alkanes as pure liquids when chain length exceeded some critical value, $n=7$ or 8 . This result was interpreted

$$
{ }_{m} K=6 B^{\prime} n \lambda M /(\varepsilon+2)^{2}\left(n^{2}+2\right)^{2} d
$$

where $B^{\prime}$ is the Kerr constant; $n$ is the refractive index; $\lambda$ is the wave length of the incident beam; $M$ is the molecular weight; $\varepsilon$ is the dielectric constant and $d$ is the density. $B^{\prime}$ is given by

$$
B^{\prime}=\left(n_{\|}-n_{\perp}\right) / \lambda E^{2}
$$

where $n_{\|}$and $n_{\perp}$ are the refractive indices parallel and perpendicular to an applied electric field of strength $E$. His ${ }^{5}$ and our ${ }^{1}$ molar Kerr constants correlate by ${ }_{m} K=K_{m} / 9$.

Stuart, et al., ${ }^{6}$ gave their results in the form of $B^{\prime}$. 
as being due to the short-range orientational order among $n$-alkane chains in the condensed phase. ${ }^{18}$ Such a deviation is not apparent in the present case.

\section{POLYOXYETHYLENE GLYCOLS AND POLYOXYETHYLENE DIMETHYL ETHERS}

The planar trans conformation of these chains is shown in Figure 2. It stands for the POEG or POEDE chain, depending on $\mathrm{R}=\mathrm{H}$ or $\mathrm{CH}_{3}$.

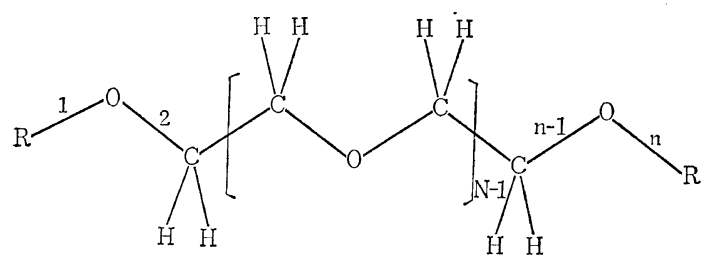

Figure 2. Poly(oxyethylene) chain in its trans planar conformation. Poly(oxyethylene glycol) or poly(oxyethylene dimethyl ether), depending on whether $\mathrm{R}=\mathrm{H}$ or $\mathrm{CH}_{3}$.

All bond angles are assumed to be tetrahedral except for $\angle \mathrm{COH}$ which is taken to be $108^{\circ} .^{30}$ Gauche angles are assumed to occur at $\pm 120^{\circ}$.

We follow Mark and Flory ${ }^{30,31}$ in models and in notations for conformational energies and statistical weights. The partition function can be expressed, according to eq 39 , as

$$
Z=\mathbf{e}_{3}{ }^{T} \mathbf{p}_{1} \mathbf{p}_{2}\left(\mathbf{p}_{a} \mathbf{p}_{b} \mathbf{p}_{c}\right)^{N-1} \mathbf{p}_{n-2} \mathbf{p}_{n-1} \mathbf{p}_{n} \mathbf{e}_{3}
$$

The statistical-weight matrices for interior bond pairs, C-C-O, C-O-C, and $\mathrm{O}-\mathrm{C}-\mathrm{C}$, are given by

$$
\mathbf{p}_{a}=\left[\begin{array}{lll}
1 & 1 & 1 \\
\sigma^{\prime} & \sigma^{\prime} & \sigma^{\prime} \omega \\
\sigma^{\prime} & \sigma^{\prime} \omega & \sigma^{\prime}
\end{array}\right]
$$

$$
\begin{aligned}
& \mathbf{p}_{b}=\left[\begin{array}{lll}
1 & 1 & 0 \\
\sigma & \sigma & 0 \\
\sigma & 0 & \sigma
\end{array}\right] \\
& \mathbf{p}_{c}=\left[\begin{array}{lll}
1 & 1 & 1 \\
\sigma & \sigma & \sigma \omega \\
\sigma & \sigma \omega & \sigma
\end{array}\right]
\end{aligned}
$$

For terminal bonds we have $\mathbf{p}_{n}=\mathbf{E}_{3}$ and

$$
\mathbf{p}_{1}=\left[\begin{array}{lll}
1 & 1 & 1 \\
1 & 1 & 1 \\
1 & 1 & 1
\end{array}\right]
$$

The remaining matrices depend on $\mathrm{R}=\mathrm{H}$ or $\mathrm{CH}_{3}$; we have for $\mathrm{H}$

$$
\begin{aligned}
& \mathbf{p}_{2}=\mathbf{p}_{n-1}=\mathbf{p}_{1} \\
& \mathbf{p}_{n-2}=\left[\begin{array}{lll}
1 & 1 & 1 \\
\sigma^{\prime} & \sigma^{\prime} & \sigma^{\prime} \\
\sigma^{\prime} & \sigma^{\prime} & \sigma^{\prime}
\end{array}\right]
\end{aligned}
$$

but for $\mathrm{CH}_{3}$

$$
\mathbf{p}_{2}=\mathbf{p}_{c}, \mathbf{p}_{n-2}=\mathbf{p}_{a}, \quad \text { and } \quad \mathbf{p}_{n-1}=\mathbf{p}_{b}
$$

In these equations $\sigma^{\prime}$ is the statistical weight of the $G$ or $G^{\prime}$ state relative to the $T$ of the bond $\mathrm{C}-\mathrm{C}$, and $\sigma$ is a similar quantity related to $\mathrm{C}-\mathrm{O}$. $\omega$ is the statistical weight related to the two-bond interaction in the $G G^{\prime}$ or $G^{\prime} G$ conformation of the bond pair C-C-O. The latter conformations are discarded for the bond pair C-O-C for a steric reason, as implied in eq $50{ }^{31}$ We assume the Boltzmann relations $\sigma=\exp \left(-E_{\sigma} / R T\right)$, etc. Estimations of these parameters were made by Mark and Flory ${ }^{30,31}$ through analysis of the unperturbed dimension, the dipole moment, and their temperature coefficients of poly(oxyethylene) and its oligomers; and by Fourche ${ }^{24}$ through analysis of the optical anisotropy of derivatives of oxyethylene; and by

Table III. Conformational energy estimates from various sources

\begin{tabular}{cccll}
\hline \multicolumn{2}{c}{ Conformational energy, kcal/mol } & & \multicolumn{1}{c}{ Author } \\
\hline$E_{\sigma}$ & $E_{\sigma^{\prime}}$ & $E_{\omega}$ & & \\
\hline $1.2-1.7$ & $-0.43--0.39$ & $0-0.34$ & Viscosity and rubber elasticity & Mark and Flory ${ }^{31}$ \\
0.9 & -0.43 & 0.34 & Dipole moment & Mark and Flory \\
$1.1 \pm 0.15$ & $-0.25 \pm 0.25$ & $0.25 \pm 0.3$ & Optical anisotropy & Fourche \\
1.1 & - & - & Infrared and Raman spectra & Wieser, et al. $^{32}$ \\
1.5 & - & - & Raman spectra & Kitagawa and Miyazawa $^{33}$ \\
\hline
\end{tabular}


others. $^{32,33}$ These results are summarized in Table III. We choose a representative set of values of the parameters for later use: $\sigma=0.156$, $\sigma^{\prime}=1.5$, and $\omega=0.22$ at $25^{\circ} \mathrm{C}$, or $E_{\sigma}=1.1 \mathrm{kcal} /$ $\mathrm{mol}, E_{\sigma^{\prime}}=-0.24 \mathrm{kcal} / \mathrm{mol}$, and $E_{\omega}=0.9 \mathrm{kcal} / \mathrm{mol}$. The values of $E_{\sigma}$ and $E_{\sigma^{\prime}}$ were selected close to those given by Fourche, ${ }^{24}$ and the value of $E_{\omega}$ was selected to correctly reproduce the experimental characteristic ratio $\left\langle r^{2}\right\rangle / n b^{2}$ of the unperturbed dimension recently reported by Beech and Booth $^{34}$ (4.805 calculated ${ }^{35}$ using the above values of $\sigma$, etc., vs. observed $4.8^{34}$ ). The above set of parameters will later be checked with dipole moments and optical anisotropies of some oligomers of oxyethylene.

Analogous to the case of $n$-alkanes, with the tetrahedral symmetray of bond angles it can be regarded that $\mathrm{C}-\mathrm{C}$ bonds were to have the effective anisotropy $\Gamma_{\mathrm{C}-\mathrm{C}}$ while the C-O bonds were to have

$$
\Gamma_{\mathrm{C}-\mathrm{O}}=\left(\alpha_{1}-\alpha_{2}\right)_{\mathrm{C}-\mathrm{O}}-\left(\alpha_{1}-\alpha_{2}\right)_{\mathrm{C}-\mathrm{H}}
$$

Experimental values of $\Gamma_{\mathrm{C}-\mathrm{o}}$ reported by various authors are included in Table $I$. Unfortunately they show considerable scattering, as in the case of $\Gamma_{\mathrm{C}-\mathrm{C}}$. Fourche ${ }^{24}$ estimated $\Gamma_{\mathrm{C}-\mathrm{O}}=$ $1.0 \AA^{3}$ from optical anisotropies of methyl ether and dioxane-1, 4. Almost the same value can

Table IV. Values of $\Gamma_{\mathrm{C}-\mathrm{O}}$ so adjusted a ${ }^{\mathrm{a}}$ as to reproduce observed 24,36 optical anisotropies of $\mathrm{CH}_{3} \mathrm{O}\left(\mathrm{CH}_{2} \mathrm{CH}_{2} \mathrm{O}\right)_{N} \mathrm{CH}_{3}$, averaged for the members $N=1-4$.

\begin{tabular}{ccll}
\hline$\sigma$ & $\sigma^{\prime}$ & $\omega$ & $\Gamma_{\mathrm{C}-\mathrm{O}}, \AA^{3}$ \\
\hline 0.223 & 1.0 & 0 & 0.981 \\
0.223 & 1.5 & 0 & 1.051 \\
0.223 & 1.5 & 0.5 & 1.077 \\
0.223 & 1.5 & 1.0 & 1.096 \\
0.223 & 2.0 & 0 & 1.102 \\
0.135 & 1.0 & 0 & 0.906 \\
0.135 & 1.5 & 0 & 0.977 \\
0.135 & 1.5 & 0.5 & 0.977 \\
0.135 & 1.5 & 1.0 & 1.012 \\
0.135 & 2.0 & 0 & 1.027 \\
0.082 & 1.0 & 0 & 0.853 \\
0.082 & 1.5 & 0 & 0.927 \\
0.082 & 1.5 & 0.5 & 0.941 \\
0.082 & 1.5 & 1.0 & 0.953 \\
0.082 & 2.0 & 0 & 0.990 \\
\hline
\end{tabular}

a $\Gamma_{\mathrm{C}-\mathrm{C}}=0.81 \AA^{3}$ is assumed. be obtained from data of optical anisotropies of POEDE with $N=1-4$, reported by Fourche. ${ }^{24,36}$ We have calculated values of $\Gamma_{\mathrm{C}-\mathrm{O}}$ which together with $\Gamma_{\mathrm{C}-\mathrm{C}}=0.81 \AA^{3}$ reproduce observed values for optical anisotropies of these compounds, for various combinations of statistical-weight parameters which are thought in totality to encompass real values. Average values of $\Gamma_{\mathrm{C}-\mathrm{O}}$ for the four compounds are given in Table IV. Use of $0.87 \AA^{3}$ instead of $0.81 \AA^{3}$ for $\Gamma_{\mathrm{C}-\mathrm{C}}$ yields values about $2 \%$ less. We see in Table IV that $\Gamma_{\mathrm{C}-\mathrm{O}}$ remains almost constant $\left(1 \AA^{3}\right)$ while statistical weights vary considerably. On this basis the value $\Gamma_{\mathrm{C}-\mathrm{O}}=1 \AA^{3}$ by Fourche ${ }^{24}$ is considered reliable and will be used in what follows.

Fourche $^{36}$ assumed $\left(\alpha_{1}-\alpha_{2}\right)_{\mathrm{O}-\mathrm{H}}$ to be equal to $\left(\alpha_{1}-\alpha_{2}\right)_{\mathrm{C}-\mathrm{H}}=0.22 \AA^{3}$, based on the fact that the C-C and C-O bond anisotropies are close: $1.31 \AA^{3}$ vs. $1.21 \AA^{3}$. We follow this convention on account of the lack of reliable evidence. Obviously, $\left(\alpha_{1}-\alpha_{2}\right)_{\mathrm{O}-\mathrm{H}}$ is significant only for very short chains.

We assume $m_{\mathrm{C}-\mathrm{C}}=m_{\mathrm{C}-\mathrm{H}}=0$. We adopt $m_{\mathrm{C}-\mathrm{O}}=1$ $\mathrm{D}$ and $m_{\mathrm{O}-\mathrm{H}}=1.7 \mathrm{D}$, the values determined by Mark and Flory ${ }^{30}$ from dipole moments of diethyl ether and ethanol.

We now check the values of the parameters given above for oligomers of oxyethylene. Use of $\Gamma_{\mathrm{C}-\mathrm{O}}=1, \Gamma_{\mathrm{C}-\mathrm{C}}=0.81,\left(\alpha_{1}-\alpha_{2}\right)_{\mathrm{O}-\mathrm{H}}=0.22$ (all in $\AA^{3}$ ), $m_{\mathrm{C}-\mathrm{O}}=1$ and $m_{\mathrm{O}-\mathrm{H}}=1.7$ (in D) and $\sigma=1.5$, yields for the dipole moment and the optical anisotropy of ethylene glycol, 2.383 D and 1.444 $\AA^{6}$, in close agreement with the experimental values $2.38 \mathrm{D}^{37}$ and $1.46 \AA^{6} .^{36}$ Use of $\sigma=0.156$ and $\omega=0.22$, in addition to those just cited above, yields for pentaethylene glycol 3.292 D and $11.097 \AA^{6}$, in comparison with the experimental values $3.42 \mathrm{D}^{37}$ and $11.35 \AA^{6} .^{36}$

Aroney, Le Fevre, and Parkins ${ }^{7}$ measured molar Kerr constants of a series of POEG with $N=4.1,6.4,18,34,78$, and 153 , in benzene at $25^{\circ} \mathrm{C}$. Their results converted to $K_{m} / N$ are plotted by circles against $N$ in Figure 5 .

We wish to refer to the so-called excludedvolume effect. It is currently argued ${ }^{38}$ that the excluded-volume effect on chain dimensions vanishes for short chains on the basis of the fact that the exponent $a$ in the Mark-HowinkSakurada relation $[\eta]=K M^{a}$ tends to 0.5 for short chains, a value theoretically predicted for 


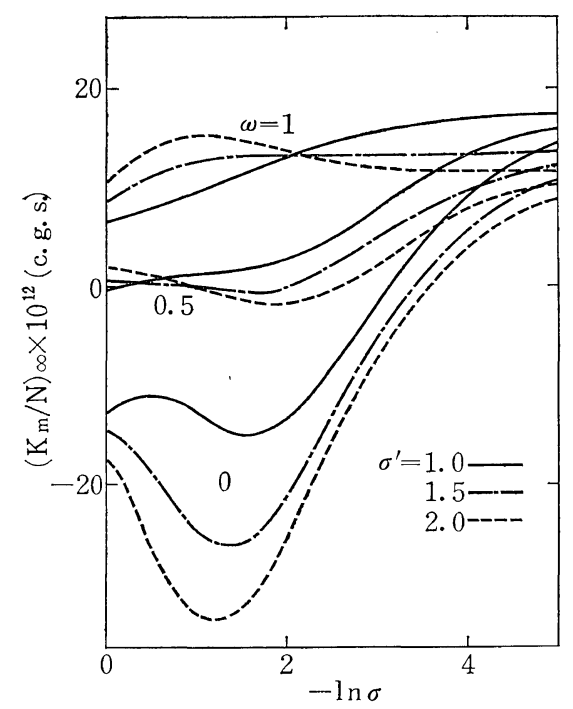

A

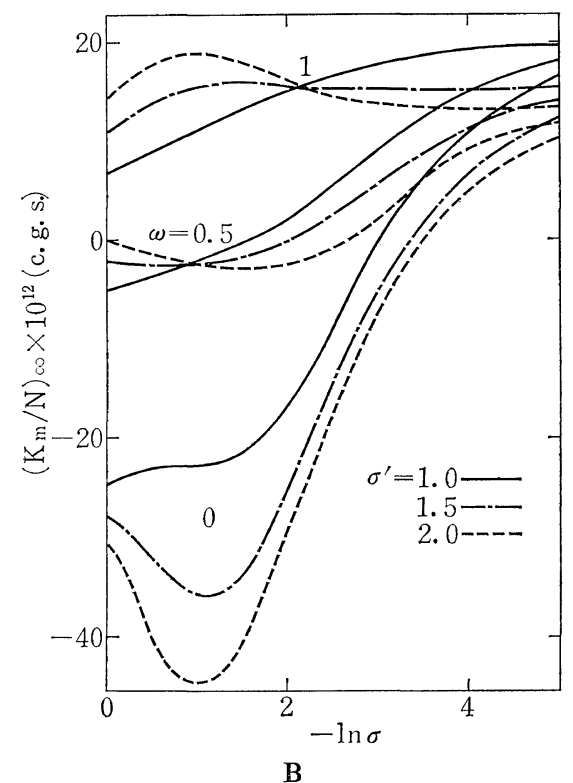

Figure 3. $\left(K_{m} / N\right)_{\infty} v s$. $-\ln \sigma$ for poly(oxyethylene)s. Parameters used are as follows: A, $\Gamma_{\mathrm{C}-\mathrm{C}}=$ $0.81 \AA^{3}, \Gamma_{\mathrm{C}-\mathrm{O}}=1 \AA^{3}$, and $m_{\mathrm{C}-\mathrm{O}}=1 \mathrm{D} ; \mathrm{B}, \Gamma_{\mathrm{C}-\mathrm{C}}=1.44$ $\AA^{3}, \Gamma_{\mathrm{C}-\mathrm{O}}=0.77 \AA^{3}$, and $m_{\mathrm{C}-\mathrm{O}}=1 \mathrm{D}$; others as indicated.

long unperturbed chains. The excluded-volume effect on chain dimensions may therefore be considered to become significant for $M$ larger than some critical value $M_{c}$ above which the deviation of $a$ from 0.5 becomes perceptible. For POEG in benzene at $25^{\circ} \mathrm{C}, M_{c} \simeq 10^{3}$ or $N_{c} \simeq$ 22, according to Rossi and Cuniberti. ${ }^{39}$ We would expect that the excluded volume has less effect on the dipole-moment term of $K_{m}$ than on dimensions, on the analogy of the case of dipole moments, ${ }^{40}$ and that it has a comparable effect on the polarizability term. ${ }^{*}$ It is found that for both POEG and POEDE the dipolemoment term always plays a more important role in $K_{m}$ than the polarizability term. In summary the excluded-volume effect does not seem very important in the present case, in particular for short chains $N<20$. Theoretical values of $K_{m} / N$ almost converge to limiting values for $N>30$ irrespective of values of the parameters (see Figure 5), whereas the observed values for $N=34,78$, and 153 are distinctly different.

We first calculated values of $\left(K_{m} / N\right)_{\infty}$, that is, $K_{m} / N$ for the infinite chain, to examine the effect of various statistical-weight parameters on $\left(K_{m} / N\right)_{\infty}$. Limiting values can be obtained by fitting finite-chain values to an asymptotic relation $K_{m}=a+b N$. Sufficiently accurate values of $b$ were obtained at $N=16-20$. Values of $\left(K_{m} / N\right)_{\infty}$ are plotted against $-\ln \sigma$ for some combinations of $\sigma^{\prime}$ and $\omega$ and for the two selected sets of effective bond anisotropies, $\Gamma_{\mathrm{C}-\mathrm{C}}=0.81$

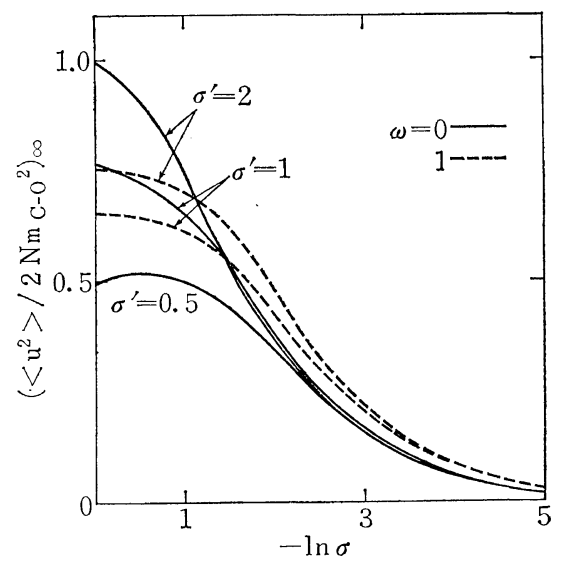

Figure 4. $\left(\left\langle u^{2}\right\rangle / 2 N m_{\mathrm{C}-\mathrm{O}}{ }^{2}\right)_{\infty}$ vs. $-\ln \sigma$ for poly(oxyethylene)s.

* The effect of the excluded volume on the dipole moment, the optical anisotropy, and the molar Kerr constant needs theoretical investigation. 


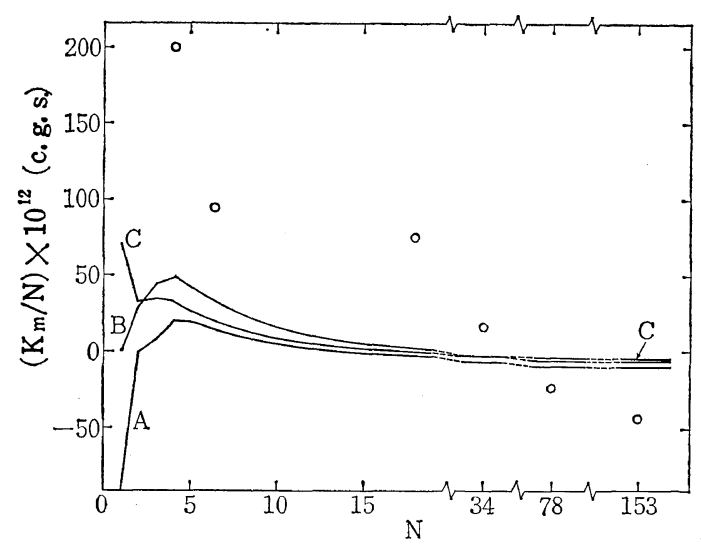

Figure 5. $K_{m} / N$ vs. $N$ for poly(oxyethylene glycol)s. Curves are calculated using the following parameters: A, $\Gamma_{\mathrm{C}-\mathrm{C}}=0.81$ and $\Gamma_{\mathrm{C}-\mathrm{O}}=1 ; \mathrm{B}, \Gamma_{\mathrm{C}-\mathrm{C}}=1.44$ and $\Gamma_{\mathrm{C}-\mathrm{O}}=0.77 ; \mathrm{C}, \Gamma_{\mathrm{C}-\mathrm{C}}=0.81$ and $\Gamma_{\mathrm{C}-\mathrm{O}}=0.23$, all in $\AA^{3}$. $\quad\left(\alpha_{1}-\alpha_{2}\right)_{\mathrm{O}-\mathrm{H}}=0.22 \AA^{3}, m_{\mathrm{C}-\mathrm{O}}=1 \mathrm{D}, m_{\mathrm{O}-\mathrm{H}}=1.7$ $\mathrm{D}, \sigma=0.156, \sigma^{\prime}=1.5$, and $\omega=0.22$. Circles are experimental data from Aroney, et al. ${ }^{7}$

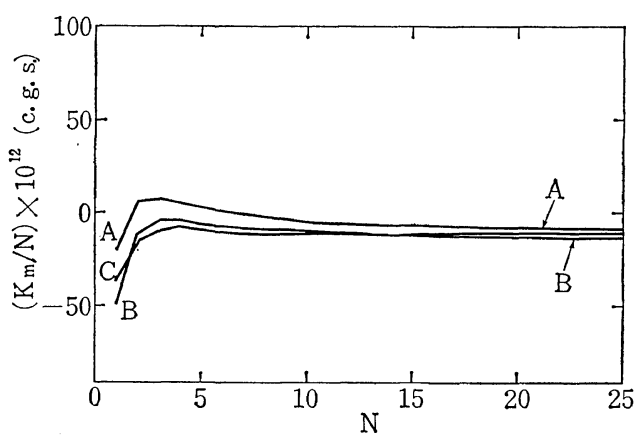

Figure 6. $K_{m} / N$ vs. $N$ for poly(oxyethylene dimethyl ether)s. Curves are calculated using the same values for parameters as in Figure 5.

and $\Gamma_{\mathrm{C}-\mathrm{O}}=1$ in Figure $3 \mathrm{~A}$, and $\Gamma_{\mathrm{C}-\mathrm{C}}=1.44$ and $\Gamma_{\mathrm{C}-\mathrm{O}}=0.77$ (all in $\AA^{3}$ ) in Figure $3 \mathrm{~B}$. From these figures it may be seen that if the negative sign of the observed values of $K_{m} / N$ for $N=78$ and 153 is taken as real and indicative of the negativity of $\left(K_{m} / N\right)_{\infty}$, this fact imposes a restriction on $\omega, i . e ., \omega<0.5$ provided $-\ln \sigma \simeq 2$. Similar plots for the dipole moment are shown in Figure 4 for the sake of comparison.

In Figure 5 we included theoretical curves for a set of statistical-weight parameters, mentioned earlier, and for three sets of bond-anisotropy parameters. The theory correctly explains the general trend of observed values, $i . e .$, a gradual decrease in $K_{m} / N$ with increasing $N$, but agreement is rather poor from the quantitative point of view. The discrepancy seems to go far beyond any uncertainties in the model and in statistical-weight and bond-anisotropy parameters, and hence does not permit adequate explanation at present.

Finally we consider POEDE. Theoretical values of $K_{m} / N$ for sets of parameters common to POEG are shown in Figure 6. They differ significantly from those for POEG only for $N<$ 20. No experimental data are available for this series.

\section{REFERENCES}

1. K. Nagai and T. Ishikawa, J. Chem. Phys., 43, 4508 (1965).

2. P. J. Flory and R. L. Jernigan, J. Chem. Phys., 48, 3823 (1968).

3. P. J. Flory, "Statistical Mechanics of Chain Molecules,' Interscience, New York, N. Y., 1969, Chapter IX.

4. K. Nagai, J. Chem. Phys., 51, 1091 (1969).

5. R. J. W. Le Fevre, "Molecular Refractivity and Polarizability," in "Advances in Physical Organic Chemistry," Vol. 3, V. Gold, Ed. Academic Press, New York, N. Y., 1965.

6. H. A. Stuart, "Landolt-Börnstein Zahlenwerte und Funktionen," 6th ed., Vol. II, Part 8, Springer-Verlag, Berlin, 1962, p 851; H. A. Stuart, "Die Struktur des Freien Moleküls," Springer-Verlag, Berlin, 1952, p 460.

7. M. Aroney, R. J. W. Le Fevre, and G. M. Parkins, J. Chem. Soc., 1960, 2890.

8. K. Nagai, J. Chem. Phys., 31, 1169 (1959).

9. P. J. Flory, Proc. Natl. Acad. Sci. U.S., 51, 1060 (1964); P. J. Flory and R. L. Jernigan, J. Chem. Phys., 42, 3509 (1965).

10. K. Nagai and T. Ishikawa, J. Chem. Phys., 45, 3128 (1966).

11. K. Nagai, J. Chem. Phys., 38, 924 (1963).

12. K. Nagai, J. Phys. Chem., 74, 3411 (1970).

13. K. Nagai, J. Chem. Phys., 47, 2052 (1967); 51, 1265 (1969).

14. C. A. J. Hoeve, J. Chem. Phys., 35, 1266 (1961).

15. K. Nagai and T. Ishikawa, J. Chem. Phys., 37, 496 (1962).

16. A. Abe, R. L. Jernigan, and P. J. Flory, J. Amer. Chem. Soc., 88, 631 (1966).

17. C. P. Smyth, "Dielectric Behavior and Structure," McGraw-Hill, New York, N. Y., 1955. 
18. K. Nagai, J. Chem. Phys., 47, 4690 (1967).

19. R. A. Sack, J. Chem. Phys., 25, 1087 (1956).

20. R. P. Smith and E. M. Mortensen, J. Chem. Phys., 32, 502, 508 (1960).

21. K. G. Denbigh, Trans. Faraday Soc., 36, 936 (1940).

22. C. Clement and P. Bothorel, Compt. Rend., 258, 4054 (1964); P. Bothorel, P. Maraval and C. Clement, ibid., 265, 1068 (1967).

23. C. W. Bunn and R. Daubeny, Trans. Faraday Soc., 50, 1173 (1954).

24. G. Fourche, J. Chim. Phys., 65, 1500 (1968).

25. C. G. Le Fevre and R. J. W. Le Fevre, J. Chem. Soc., 1956, 3549.

26. R. J. W. Le Fevre, B. J. Orr, and G. L. D. Ritchie, J. Chem. Soc., Sect. B, 1966, 273.

27. T. Ishikawa and K. Nagai, J. Polym. Sci., Part A-2, 7, 1123 (1969).

28. Landolt-Börnstein, "Physikalisch-Chemische Tabellen und Ergänzungsband," W. A. Roth and K. Scheel, Ed., Springer-Verlag, Berlin, $1923-36$.
29. K. Nagai, J. Chem. Phys., 49, 4212 (1968).

30. J. E. Mark and P. J. Flory, J. Amer. Chem. Soc., 88, 3702 (1966).

31. J. E. Mark and P. J. Flory, J. Amer. Chem. Soc., 87, 1415 (1965).

32. H. Wieser, W. G. Laidlaw, P. J. Krueger, and H. Fuhrer, Spetrochim. Acta, 24A, 1055 (1968).

33. T. Kitagawa and T. Miyazawa, Bull. Chem. Soc. Japan, 41, 1976 (1968).

34. D. R. Beech and C. Booth. J. Polym. Sci., Part A-2, 7, 575 (1969).

35. T. Ishikawa, to be published.

36. G. Fourche, J. Chim. Phys., 66, 320 (1969).

37. T. Uchida, Y. Kurita, J. Koizumi, and M. Kubo, J. Polym. Sci., 21, 313 (1956).

38. For example, U. Bianchi and A. Peterlin, $J$. Polym. Sci., Part A-2, 6, 1759 (1968), and papers cited therein.

39. C. Rossi and C. Cuniberti, Polym. Letters, 2, 681 (1964).

40. J. Marchal and H. Benoit, J. Chim. Phys., 52, 818 (1955); J. Polym. Sci., 23, 223 (1957). 